\title{
Magnetotransport Properties of Epitaxial Ge/AIAs Heterostructures Integrated on GaAs and Silicon
}

\author{
Mantu K. Hudait, ${ }^{\dagger}{ }^{\dagger}$ Michael Clavel, ${ }^{\dagger}$ Patrick S. Goley, ${ }^{\dagger}$ Yuantao Xie, ${ }^{\dagger}$ and Jean J. Heremans ${ }^{\ddagger}$ \\ ${ }^{\dagger}$ Advanced Devices \& Sustainable Energy Laboratory (ADSEL), Bradley Department of Electrical and Computer Engineering, \\ Virginia Tech, Blacksburg, Virginia 24061, United States \\ ${ }^{\ddagger}$ Department of Physics, Virginia Tech, Blacksburg, Virginia 24061, United States
}

ABSTRACT: The magnetotransport properties of epitaxial $\mathrm{Ge} / \mathrm{AlAs}$ heterostructures with different growth conditions and substrate architectures have been studied under $\pm 9 \mathrm{~T}$ magnetic field and at $390 \mathrm{mK}$ temperature. Systematic mobility measurements of germanium (Ge) epilayers grown on $\mathrm{GaAs}$ substrates at growth temperatures from 350 to $450{ }^{\circ} \mathrm{C}$ allow us to extract a precise growth window for device-quality $\mathrm{Ge}$, corroborated by structural and morphological properties. Our results on Si substrate using a composite metamorphic AlAs/ GaAs buffer at $400{ }^{\circ} \mathrm{C}$ Ge growth temperature, show that the
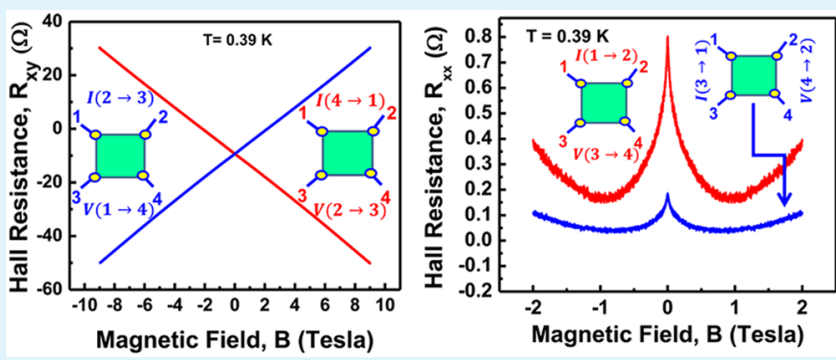

$\mathrm{Ge} / \mathrm{AlAs}$ system can be tailored to have a single carrier transport while keeping the charge solely in the Ge layer. Single carrier transport confined to the Ge layer is demonstrated by the weak-localization quantum correction to the conductivity observed at low magnetic fields and $390 \mathrm{mK}$ temperature. The weak localization effect points to a near-absence of spin-orbit interaction for carriers in the electronically active layer and is used here for the first time to pinpoint Ge as this active layer. Thus, the epitaxial Ge grown on Si using AlAs/GaAs buffer architecture is a promising candidate for next-generation energy-efficient fin field-effect transistor applications.

KEYWORDS: germanium, transport, epitaxy, molecular beam epitaxy, heterostructure

\section{INTRODUCTION}

Germanium $(\mathrm{Ge})$ is an attractive material due to its higher electron and hole mobilities than silicon $(\mathrm{Si})$ for future generation low-power and high-speed nanoscale logic transistors. In the last several decades, transistors using Ge directly deposited on $\mathrm{Si}^{1,2} \mathrm{Ge}$ on $\mathrm{Si}$ using graded $\mathrm{SiGe}$ buffer, ${ }^{3,4} \mathrm{Ge}$-oninsulator-on-Si (GeOI) by bonding, ${ }^{5}$ compressively strained $\mathrm{Ge}$ in a quantum well (QW) configuration on $\mathrm{Si}^{6}{ }^{6}$ and bulk $\mathrm{Ge},{ }^{7}$ all have been studied. Yet these methods have not achieved higher valence band and conduction band offsets for carrier confinement. Very recently, epitaxial Ge heterogeneously integrated on Si using composite metamorphic AlAs/GaAs buffers ${ }^{8,9}$ obtained by molecular beam epitaxy (MBE) illustrated a promising path to understand the effect of growth temperature, the role of an AlAs buffer layer underneath the Ge layer and their effect on the magnetotransport properties of epitaxial Ge. It has been well-documented that the large bandgap semiconductor buffer layers such as GaAs, InAlAs, ${ }^{10,11}$ or oxide buffer layer ${ }^{12}$ can be considered to eliminate the parallel conduction either from the substrate or through the buffer layer to the active device layer of interest. For example, in a metamorphic InGaAs QW transistor structure on $\mathrm{Si}$, the composite $\mathrm{GaAs} / \mathrm{In}_{x} \mathrm{Al}_{1-x} \mathrm{As}$ buffer eliminates the parasitic conduction to the active InGaAs channel. $^{10,11}$ In order to achieve a device-quality epitaxial Ge layer on large bandgap buffer, it is necessary to understand the role of the buffer layer, the growth temperature, and the growth pause that provides the film quality.
The temperature-dependent mobility as measured by the van der Pauw technique and subsequent analysis by the quantitative mobility spectrum (QMSA) are often used to determine the (1) channel mobility, (2) parallel conduction to active channel, and (3) the carrier freeze-out for high mobility III-V materials. ${ }^{10,11,13,14}$ However, the QMSA method fails for materials with mobility below $1000 \mathrm{~cm}^{2} /(\mathrm{V} \mathrm{s})$. Therefore, it is necessary to find an alternative way to determine the mobility and carrier density contributed solely by the Ge layer grown on a large bandgap material, such as AlAs, where carriers are confined within the Ge layer. Furthermore, a well-controlled heterointerface between the epitaxial $\mathrm{Ge}$ and the large bandgap AlAs buffer layer is crucial to realize high-performance nanoscale $\mathrm{Ge}$ transistors. Here, we report on the growth by $\mathrm{MBE}$ of a series of $\mathrm{Ge} / \mathrm{AlAs} / \mathrm{GaAs}$ structures as well as a $\mathrm{Ge}$ / $\mathrm{AlAs} / \mathrm{GaAs} / \mathrm{Si}$ structure and their characterization. In particular, we discuss the effect of (1) the AlAs buffer layer, (2) the duration of the growth pause, (3) the growth temperature, (4) the different annealing temperatures, and (5) the reliability of repeated mobility measurements from 90 to $315 \mathrm{~K}$ of the $\mathrm{Ge}$ layers. The structures were grown using two solid-source $\mathrm{MBE}$ chambers, connected via an ultrahigh vacuum transfer chamber. We also note that the in situ growth process of Ge following

Received: June 29, 2015

Accepted: September 28, 2015 
the large bandgap AlAs buffer is mandatory since the AlAs layer is prone to oxidize once removed from the ultrahigh vacuum chamber prior to epitaxial Ge layer growth. The Ge/AlAs/ $\mathrm{GaAs} / \mathrm{Si}$ structure was characterized at low temperatures (down to $390 \mathrm{mK}$ ) under magnetic fields up to $9 \mathrm{~T}$ to investigate the carrier transport behavior inside the Ge layer. The magnetotransport measurements demonstrate single carrier confinement inside the Ge layer. Furthermore, the Ge/AlAs/GaAs/Si structure exhibited weak-localization, pointing to a near absence of spin-orbit interaction. This observation in turn suggests that only carriers within the Ge layer contribute to transport. The weak localization quantum correction to the conductivity was obtained by careful sweeps of the magnetic field at the lowest measurement temperatures. Furthermore, X-ray analysis was performed to ascertain interface quality, surface morphology was visualized by atomic force microscopy (AFM), and defect/ interface properties were evaluated using cross-sectional transmission electron microscopy (TEM), all to establish pathways for $\mathrm{Ge}$ based materials and their device structures.

\section{RESULTS AND DISCUSSION}

Structural Analysis of Epitaxial Ge on GaAs with AIAs Buffer. Figure 1 shows the schematic of layer structures

\begin{tabular}{|c|c|}
\hline $\begin{array}{c}80 \mathrm{~nm} \mathrm{Ge} \\
\left(350^{\circ} \mathrm{C}, 400^{\circ} \mathrm{C}, 450^{\circ} \mathrm{C}\right)\end{array}$ & $\begin{array}{c}240 \mathrm{~nm} \mathrm{Ge} \\
\left(400^{\circ} \mathrm{C}\right)\end{array}$ \\
\hline $170 \mathrm{~nm}$ AlAs & $170 \mathrm{~nm}$ AlAs \\
\hline $250 \mathrm{~nm}$ GaAs & $2.0 \mu \mathrm{m} \mathrm{GaAs}$ \\
\hline$(100) / 2^{\circ} \mathrm{GaAs} \quad$ (a) & $(100) / 6^{\circ} \mathrm{Si}$ \\
\hline
\end{tabular}

Figure 1. Schematic of epitaxial Ge layers grown on (a) GaAs and (b) $\mathrm{Si}$ substrates, respectively. Ge epitaxial layers were grown at 350, 400, and $450{ }^{\circ} \mathrm{C}$ on GaAs substrate, and at $400{ }^{\circ} \mathrm{C}$ on Si substrate.

studied in this work. The $80 \mathrm{~nm}$ epitaxial Ge layers were grown on semi-insulating $(100) / 2^{\circ}$ offcut GaAs substrates at growth temperatures of 350,400 , and $450{ }^{\circ} \mathrm{C}$ with the growth rate of approximately $25 \mathrm{~nm} / \mathrm{h}$. The growth temperature in this study refers to thermocouple temperature during growth. The low growth rate was selected to prevent interdiffusion at the $\mathrm{Ge}$ / AlAs heterointerface. ${ }^{8,9}$ The $240 \mathrm{~nm}$ Ge layer was grown on (100) $/ 6^{\circ}$ Si substrate using the composite metamorphic AlAs/ GaAs buffer shown in Figure 1b. The detailed material analysis of $\mathrm{Ge}$ on $\mathrm{Si}$ using AlAs/GaAs buffer was reported earlier. ${ }^{8}$ The structural quality and the relaxation state of each $\mathrm{Ge}$ layer structure on GaAs were evaluated using high-resolution (004) $\mathrm{X}$-ray rocking curves as shown in Figure 2. The peak positions of $\mathrm{Ge}$, AlAs, and GaAs substrate are clearly visible in these figures. The angular separation $\Delta \theta$ between the (004) diffraction peaks of GaAs and $\mathrm{Ge}$ resulting from the difference in lattice plane spacing $\Delta d / d$ can also provide the microstructural quality. Both the AlAs and Ge peak positions with respect to the GaAs substrate attest to the lattice matched nature of the layer structure, as expected. The full width at halfmaximum (FWHM) of Ge layers were found to be 50, 51, and 47 arcsec for the growth temperatures of 350,400 , and $450{ }^{\circ} \mathrm{C}$, respectively. The $\mathrm{X}$-ray analysis thus suggests that there is a wide range of growth temperature window for the epitaxial $\mathrm{Ge}$ layer. However, the higher growth temperature must be avoided to minimize the interdiffusion of $\mathrm{Ga}$, As, and $\mathrm{Ge}$ atoms at the heterointerface.

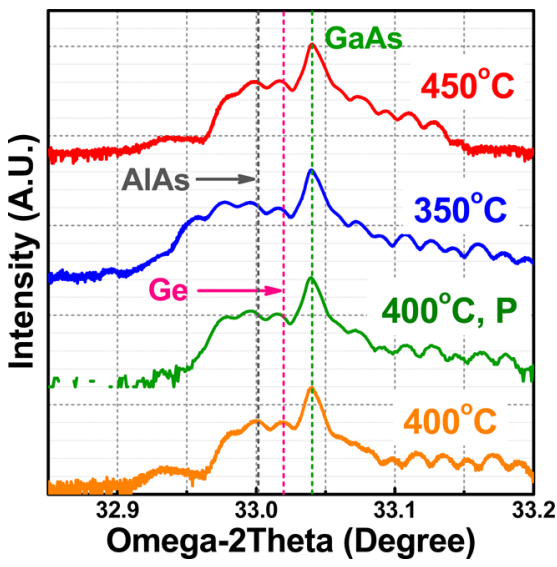

Figure 2. X-ray rocking curves for the $80 \mathrm{~nm}$ Ge layer grown on (100) $/ 2^{\circ} \mathrm{GaAs}$ substrates at 350,400 , and $450{ }^{\circ} \mathrm{C}$, respectively. The Pendellösung oscillations from these samples show the superior epitaxial crystalline quality. The X-ray curves were shifted vertically for clarity. The " $\mathrm{P}$ " represents the long growth pause prior to Ge layer growth.

The structure was further investigated by AFM to quantify the surface roughness and other growth- related defects. Figure 3 shows the AFM surface morphology of the epitaxial Ge layer grown at three growth temperatures on GaAs substrates using AlAs buffer layers without any long growth pause prior to the Ge layer growth. The AFM data reveals a root-mean-square (rms) roughness of $\sim 1.8-2.5 \mathrm{~nm}$ over $20 \times 20 \mu \mathrm{m}$ for the Ge, which is about 5 times higher than the Ge layer grown on (100) $/ 6^{\circ}$ GaAs substrate using MBE. ${ }^{15}$ The anticipated uniform and low surface roughness is an indication of highquality two-dimensional epitaxy of Ge on AlAs/GaAs. The increase in surface roughness could be due to the growth of the AlAs layer on GaAs. The same growth rate $(25 \mathrm{~nm} / \mathrm{h})$ and growth temperature $\left(400{ }^{\circ} \mathrm{C}\right)$ exhibited a surface roughness of about $0.38 \mathrm{~nm}$ for the Ge grown on (100)/6 ${ }^{\circ} \mathrm{GaAs}$ substrate. ${ }^{15}$ This is also consistent with reflection high energy electron diffraction (RHEED) observation during growth, which displayed a streakier $(2 \times 2)$ surface reconstruction pattern for Ge. ${ }^{15}$

During MBE growth, the active device layer is typically grown after a time pause of variable duration following growth of underlying layers. To understand the effect of a long growth pause prior to the growth of the Ge layer on the structural and electrical transport properties of the Ge layer, we kept the AlAs/GaAs sample under a vacuum of $\sim 10^{-9}$ Torr at $150{ }^{\circ} \mathrm{C}$ temperature inside the MBE chamber for about $12 \mathrm{~h}$ after the growth of AlAs, prior to the growth of the Ge layer. Figure 4 shows the AFM surface morphology of the epitaxial Ge layers on $(100) / 2^{\circ} \mathrm{GaAs}$ substrate grown at $400{ }^{\circ} \mathrm{C}$ with the growth pause. The measured surface roughness was $\sim 2.21 \mathrm{~nm}$, similar to the Ge layer grown at $400{ }^{\circ} \mathrm{C}$ without long growth pause ( $\mathrm{rms}$ roughness of $\sim 2.25 \mathrm{~nm}$ ), as shown in Figure $3 \mathrm{~b}$. The surface roughness of Ge layers with and without the growth pause after the growth of AlAs layer, are almost identical. The $\mathrm{X}$-ray analysis of this structure is shown in Figure 2. The electrical transport properties of these Ge layers would provide information whether the long growth pause prior to the $\mathrm{Ge}$ layer impacts the carrier mobility and ultimately the device properties. The insight will help device and process engineers by providing flexibility for designing Ge-based nanoscale transistor structures where a long growth pause in the process 

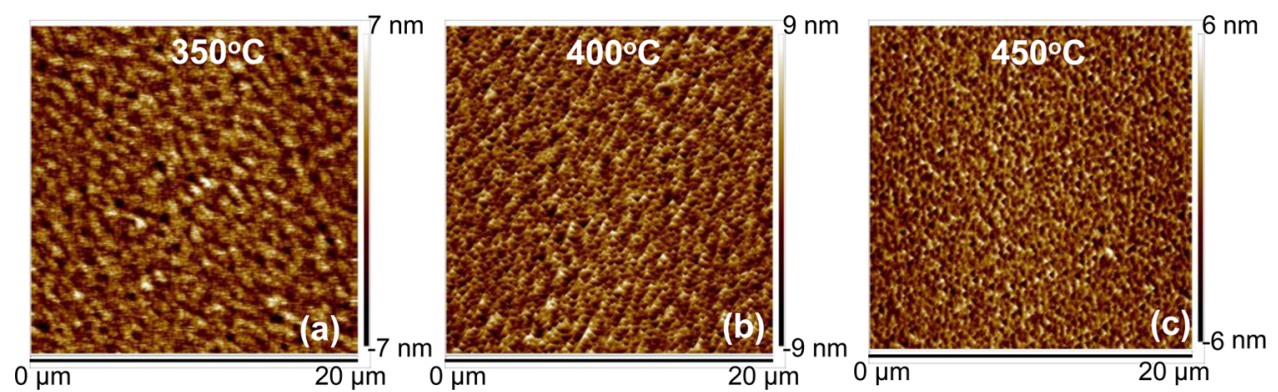

Figure 3. Surface morphology of the epitaxial Ge layers grown at three different growth temperatures on $(100) / 2^{\circ}$ GaAs substrates. The measured surface roughness are in the range of $1.8-2.5 \mathrm{~nm}$.

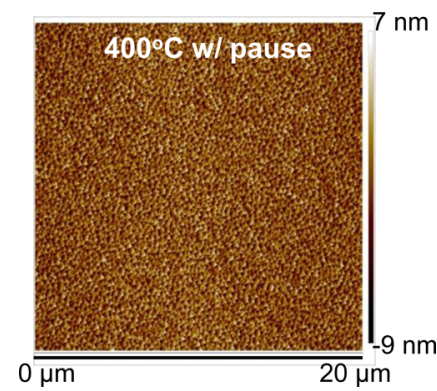

Figure 4. Surface morphology of the epitaxial Ge layer on $(100) / 2^{\circ}$ GaAs substrate grown at $400{ }^{\circ} \mathrm{C}$ with $12 \mathrm{~h}$ growth pause after the AlAs layer growth. The measured surface roughness was $2.21 \mathrm{~nm}$.

cannot be avoided. Table 1 shows the summary of the results obtained from these samples.

Table 1. Summary of Growth Parameter, Structural Analysis, And Electrical Transport Parameters of Ge Layers Grown on GaAs Substrates Presented in This Study

\begin{tabular}{|c|c|c|c|c|c|}
\hline \multicolumn{2}{|c|}{$\begin{array}{l}\text { Ge growth } \\
\text { temperature } \\
\left({ }^{\circ} \mathrm{C}\right)\end{array}$} & \multirow{2}{*}{ 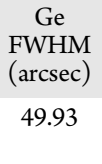 } & \multirow{2}{*}{$\begin{array}{c}\text { RMS } \\
\text { roughness } \\
(\mathrm{nm})\end{array}$} & \multirow{2}{*}{ 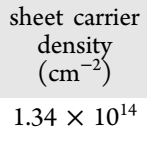 } & \multirow{2}{*}{$\begin{array}{c}\begin{array}{c}\text { electron } \\
\text { mobility } \\
\left(\mathrm{cm}^{2} /(\mathrm{V} \mathrm{s})\right.\end{array} \\
202.84\end{array}$} \\
\hline 350 & $\begin{array}{l}\text { no } \\
\text { pause }\end{array}$ & & & & \\
\hline 400 & $\begin{array}{l}\text { no } \\
\text { pause }\end{array}$ & 50.98 & 2.55 & $2.84 \times 10^{13}$ & 280.44 \\
\hline 400 & pause & 49.10 & 2.21 & $5.86 \times 10^{13}$ & 252.05 \\
\hline 450 & pause & 47.23 & 1.78 & $2.07 \times 10^{13}$ & 202.75 \\
\hline
\end{tabular}

The structural quality and the defect properties of the $80 \mathrm{~nm}$ $\mathrm{Ge} / 170 \mathrm{~nm}$ AlAs/GaAs and $240 \mathrm{~nm} \mathrm{Ge} / 170 \mathrm{~nm} \mathrm{AlAs} / 2.2 \mu \mathrm{m}$
$\mathrm{GaAs} / \mathrm{Si}$ structures were examined by cross-sectional TEM. Figure 5 shows a typical cross-sectional bright field TEM micrograph of the $\mathrm{Ge} / \mathrm{AlAs} / \mathrm{GaAs}$ and $\mathrm{Ge} / \mathrm{AlAs} / \mathrm{GaAs} / \mathrm{Si}$ structure, respectively, showing the interfaces between $\mathrm{Ge}$ and AlAs as well as AlAs and GaAs. The layer structure shown in Figure 5 was grown at $400{ }^{\circ} \mathrm{C}$ without long growth pause. The image in Figure 5 shows a high contrast at each heterointerface and the $\mathrm{Ge} / \mathrm{AlAs} / \mathrm{GaAs}$ structure is lattice matched, as expected since the lattice constants of Ge, AlAs, and GaAs are almost identical (the lattice mismatch between $\mathrm{Ge}$ and $\mathrm{GaAs}$ is $0.07 \%$ ). The lattice matched nature of the Ge layer revealed by the TEM micrograph is consistent with the results from the $\mathrm{X}$-ray analysis above, demonstrating the significant achievement toward correlated synthesis-structureproperty behavior. We also note that the AlAs layer will provide carrier confinement inside the Ge layer via the large band offsets of Ge/AlAs. ${ }^{8}$ A sufficiently high barrier for holes and electrons is indeed desired for carrier confinement. Further, the AlAs layer can serve as an etch stop layer when fabricating nanoscale Ge transistors.

Electrical Transport Properties. Effect of Growth Temperature and Growth Pause on Ge. To further investigate the quality of $\mathrm{Ge}$ layers grown at different temperatures, we measured the carrier mobility of the Ge films using electronic transport measurements in the van der Pauw configuration over a temperature range of $90-315 \mathrm{~K}$. The transport measurements assess the quality and the carrier density in the semiconductor layers, with the mobility as an important figure-of-merit. Additionally, the mobility and the sheet carrier density obtained as a function of temperature are important design parameters for the next-generation nanoscale transistors. It is important that the carrier freeze-out be minimal at lower temperature and that transport in the layers be dominated by a single carrier.
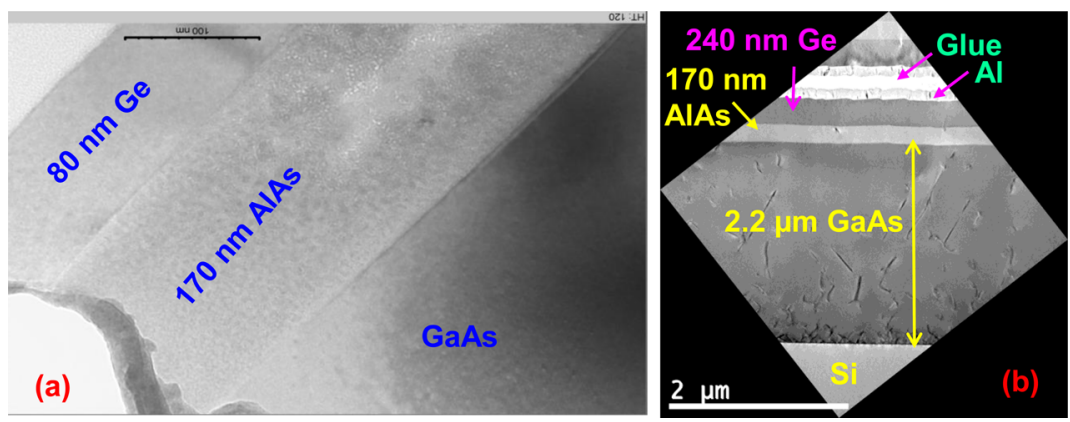

Figure 5. (a) Cross-sectional TEM micrograph of $80 \mathrm{~nm}$ Ge grown on GaAs substrate using a $170 \mathrm{~nm}$ AlAs buffer layer. The granular spots in some areas of AlAs layer are due to the damage during ion milling process. (b) Cross-sectional TEM micrograph of the Ge/AlAs/GaAs/Si structure. The TEM measurement was performed after depositing the aluminum metal. 
Figure 6 shows the electron mobility and sheet carrier density as a function of temperature in Ge layers grown at different
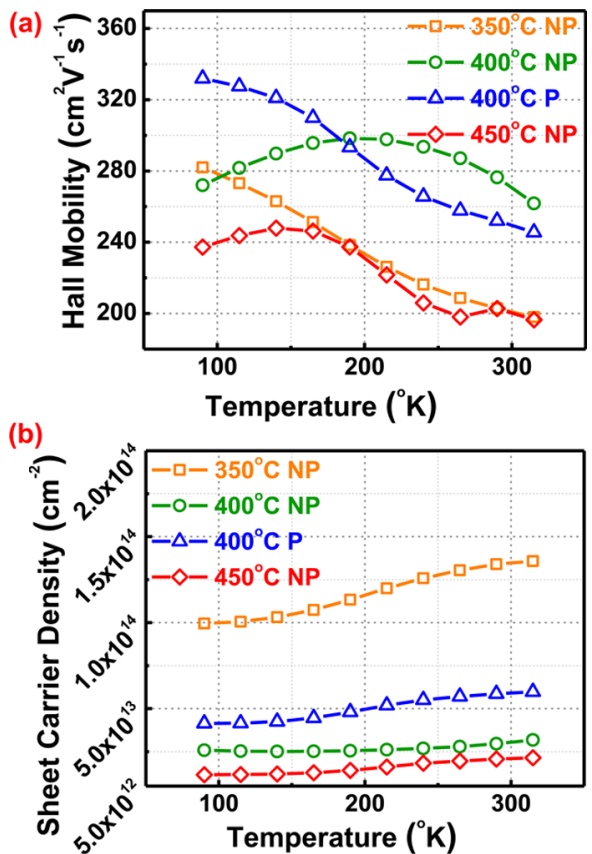

Figure 6. (a) Hall mobility and (b) sheet carrier density as a function of temperature in Ge layers grown at 350,400 , and $450{ }^{\circ} \mathrm{C}$, respectively. Ge layers were grown with without pause (NP) and with a long growth pause $(\mathrm{P})$.

temperatures. The mobility at $290 \mathrm{~K}$ was $\sim 280 \mathrm{~cm}^{2} /(\mathrm{V} \mathrm{s})$ for the sample grown at $400{ }^{\circ} \mathrm{C}$ compared to $\sim 200 \mathrm{~cm}^{2} /(\mathrm{V} \mathrm{s})$ for samples grown at 350 and $450{ }^{\circ} \mathrm{C}$ without any long growth pause. However, at $290 \mathrm{~K}$ the sample grown at $400{ }^{\circ} \mathrm{C}$ with long growth pause exhibited a mobility value of about $255 \mathrm{~cm}^{2} /$ (V s). The sheet carrier density at $290 \mathrm{~K}$ is about $3 \times 10^{13} \mathrm{~cm}^{-2}$ for a $400{ }^{\circ} \mathrm{C}$ growth temperature without growth pause. The lower mobilities at both 350 and $450{ }^{\circ} \mathrm{C}$ growth temperatures measured at $290 \mathrm{~K}$ rule out the possibility of growing highquality $\mathrm{Ge}$ epitaxial layers on GaAs at these temperatures. However, these Ge layers exhibited similar X-ray FWHM and surface roughness as the sample grown at $400{ }^{\circ} \mathrm{C}$ (between the results from structural analysis and electrical transport properties, the electrical transport properties should be given preference). The complex dependences of mobility on temperature in Figure 6a are due to competing effects of phonon scattering, scattering on Coulombic impurities and dislocations, and interface scattering at the Ge/AlAs heterointerface. It is interesting to compare the two samples grown at $400{ }^{\circ} \mathrm{C}$. Above $180 \mathrm{~K}$, the mobility is higher for the sample grown without growth pause than for the sample grown with growth pause and slowly increases with decreasing temperature. Below $180 \mathrm{~K}$, the mobility in the sample without growth pause decreases with decreasing temperature, whereas the mobility in the sample with growth pause keeps increasing. Of the competing scattering mechanisms, phonon scattering has the most pronounced temperature dependence. Phonon scattering increases with increasing temperature. Phonon-limited scattering will thus lead to a mobility decreasing with increasing temperature. A phonon-limited mobility denotes a good sample quality, since it implies other scattering mechanisms, originating in sample defects, do not dominate. The temperature dependence of the mobility in the sample with growth pause indicates just such phonon-limited behavior, indicative of improved quality. The lower mobility from 180 to $315 \mathrm{~K}$ for the sample with growth pause (compared to without), could be due to the effect of the complex balance between phonon scattering, and the other scattering mechanisms, particularly interface scattering at $\mathrm{Ge} / \mathrm{AlAs}$ heterointerface. The sheet carrier densities do not change substantially over temperatures from 90 to $315 \mathrm{~K}$ for all samples. These results suggest an ideal growth temperature of $400{ }^{\circ} \mathrm{C}$ for the Ge layer, omitting the long growth pause. The sheet carrier density and the electron mobility of these samples measured at $290 \mathrm{~K}$ are also summarized in Table 1.

Effect of Forming Gas Annealing on Ge/AIAs/GaAs. To investigate the effect of forming gas annealing on the mobility and the sheet carrier density of a Ge layer grown at $400{ }^{\circ} \mathrm{C}$ without growth pause, we annealed a van der Pauw sample for different durations but at same annealing temperature of 350 ${ }^{\circ} \mathrm{C}$ under forming gas. After each measurement, the sample was annealed in forming gas $\left(95 \% \mathrm{~N}_{2} / 5 \% \mathrm{H}_{2}\right)$ for a prescribed time. Figure 7 shows the mobility and sheet carrier density of the Ge
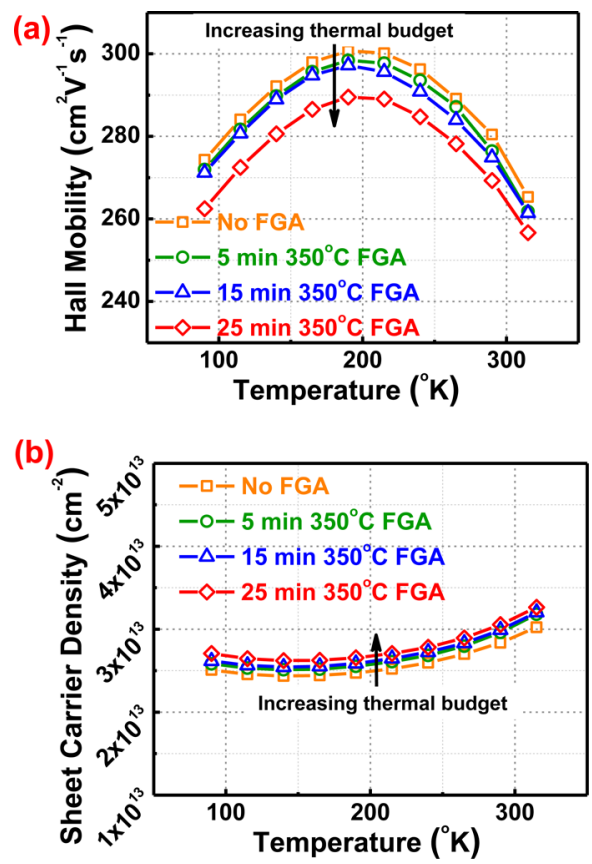

Figure 7. (a) Hall mobility and (b) sheet carrier density as a function of temperatures in Ge layers grown at $400{ }^{\circ} \mathrm{C}$ without long growth pause under different forming gas annealing (FGA) time at fixed annealing temperature of $350{ }^{\circ} \mathrm{C}$, respectively.

epilayer for annealing durations of $5,15,25 \mathrm{~min}$, and without annealing. The $15 \mathrm{~min}$ annealing duration was a cumulative time of a 5 min annealing followed by a 10 min annealing. After $15 \mathrm{~min}$ annealing and measurement, the sample was further annealed for another $10 \mathrm{~min}$ ( $25 \mathrm{~min}$ total). In each case, the Hall mobility measurement was performed from 90 to $315 \mathrm{~K}$. The shape of the mobility vs temperature graph is almost identical for different annealing durations at this fixed annealing temperature, while the mobility value decreases for longer annealing duration. The decrease is attributed to the effect of interface intermixing, creating defects within the Ge film. The sheet carrier density increases with annealing time, as shown in Figure $7 \mathrm{~b}$. Therefore, there exists a need to carefully balance the 
annealing duration and ultimately the total thermal budget for the epitaxial $\mathrm{Ge}$ layer, such that device fabrication avoids mobility degradation. It is interesting to note that the repeated thermal annealing and temperature cycle measurements from 90 to $315 \mathrm{~K}$ (low-temperature thermal cycle or cryogenic stress) on the same Hall sample have minimal effect on the carrier mobility and density, indicating that the mobility of this structure is not affected by the cryogenic stress.

Effect of Forming Gas Annealing on Epitaxial Ge on Si Using AlAs/GaAs Buffer. Results on mobility measurements on the epitaxial Ge grown on Si using large bandgap AlAs/GaAs buffer layers were provided in earlier work. ${ }^{8}$ In order to understand the effect of annealing in forming gas on the mobility and carrier density, a van der Pauw sample was repeatedly annealed in forming gas and measured over the temperature range of $90-315 \mathrm{~K}$, as described above. One purpose of these measurements is to understand the effect of hydrogen on the passivation of the shallow donors and the concomitant effect on carrier density. These experiments also allow us to ascertain the reliability of repeated mobility measurements data after cryogenic stressing on this sample. During the repeated measurements, the sample might generate a crack due to the thermal mismatch of the Ge/III-V layer and the $\mathrm{Si}$ substrate by the differences in thermal expansion in the aforementioned structure. Figure 8 shows the mobility and the

(a)
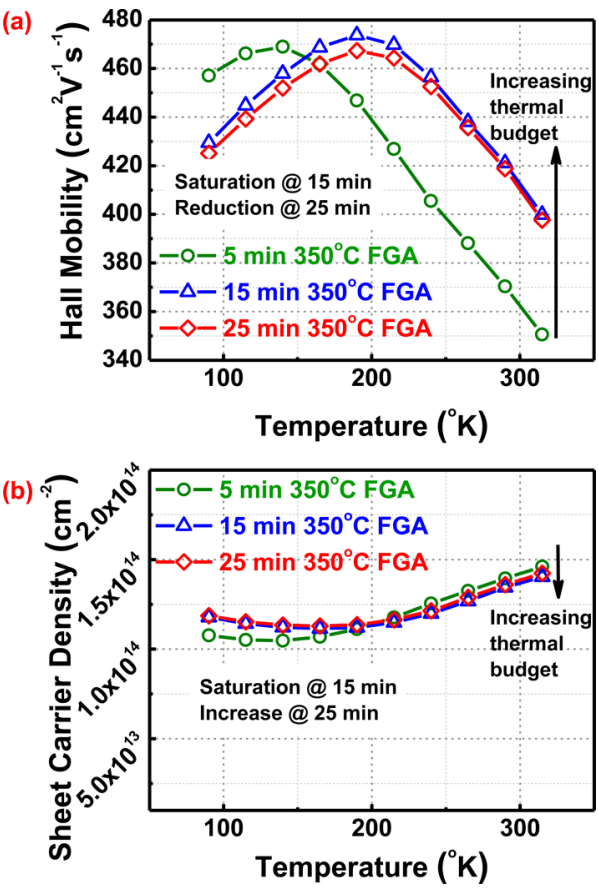

Figure 8. (a) Mobility and (b) sheet carrier density as a function of temperature in the Ge layer grown at $400{ }^{\circ} \mathrm{C}$ under different forming gas annealing FGA time, respectively, on Si using a composite AlAs/ GaAs buffer layer.

carrier density as a function of measurement temperature and forming gas annealing conditions. One can find from Figure 8a that the mobility value increased from 370 to $420 \mathrm{~cm}^{2} /(\mathrm{V} \mathrm{s})$ at $290 \mathrm{~K}$ when the sample was annealed for an additional $10 \mathrm{~min}$. This increase in mobility is attributed to the reduction of the point defects by the thermal annealing. However, the further forming gas annealing by an additional 10 min reduces the mobility, similar to Figure $7 \mathrm{a}$. Thus, a crucial total thermal budget exists for the transport properties of Ge layer either grown on $\mathrm{GaAs}$ or on $\mathrm{Si}$ substrates. The carrier density is almost constant over temperature for different annealing temperatures, suggesting that the carriers are confined to the Ge layer, with the AlAs layer acting as a parallel conduction blocking barrier over this temperature range. Furthermore, comparing the electron mobility of Ge layer grown on $\mathrm{Si}$ as well as on GaAs, one can find that the electron mobility is higher on $\mathrm{Si}$ substrate than on GaAs substrate. One might expect better crystalline quality of $\mathrm{Ge}$ on $\mathrm{GaAs}$ than on $\mathrm{Si}$ and hence higher electron mobility. However, the mobility may not correlate one-on-one with the crystal quality, and optimization of mobility is the ultimate aim. Depending on the scattering mechanism induced by crystal defects, the mobility may not severely be affected. For instance, small-angle scattering mechanisms, as due to extended Coulombic scattering potentials, will not much affect mobility since small-angle scattering is ineffective at diffusing forward momentum. We presume that the lower crystal quality obtained on $\mathrm{Si}$ still affords a higher mobility since the scattering mechanisms induced by the lower crystal quality can be ineffective at reducing mobility, whereas effective scattering mechanisms have been reduced.

Magnetotransport Measurement of Epitaxial Ge on $\mathrm{Si}$ using AlAs/GaAs Buffer. To confirm the single carrier conduction,we performed magnetotransport measurements at $390 \mathrm{mK}$ and over $\pm 9 \mathrm{~T}$ magnetic field applied normally to a sample in the van der Pauw geometry. Figure 9a shows the antisymmetric component of the Hall resistance (off-diagonal component $\mathrm{R}_{\mathrm{xy}}$ ) up to $\pm 9 \mathrm{~T}$ magnetic field with the different configurations of current and voltage contacts as depicted schematically in the insets for the van der Pauw geometry. The Hall resistance $R_{x y}$ is linear up to high $B$ in both the field directions and shows minimal intermixing with the symmetric component of Hall resistance $\mathrm{R}_{x x}$. The linearity in $B$ indicates single carrier conduction in the Ge layer. According to the linear slope, the Hall mobility measured at this temperature yielded a carrier density of $N_{B}=1.42 \times 10^{14} \mathrm{~cm}^{-2}$ and an electron mobility of $\mu_{B}=392 \mathrm{~cm}^{2} /(\mathrm{V} \mathrm{s})$, respectively, which is in agreement with the measurements depicted in Figure $8 \mathrm{a}, \mathrm{b}$. With a different contact configuration as depicted in Figure 9a, also yielding a linear slope, very nearly the same carrier density is obtained. Assuming one dominant carrier for conduction, at $390 \mathrm{mK}$ it is found that the sheet resistance is $112.72 \Omega / \square$, the sheet carrier density $1.42 \times 10^{14} \mathrm{~cm}^{-2}$, and the mobility 392 $\mathrm{cm}^{2} /(\mathrm{V} \mathrm{s})$ for the epitaxial Ge on Si using AlAs/GaAs buffer.

Magnetotransport measurements at low temperatures (below $\sim 1 \mathrm{~K}$, here at $390 \mathrm{mK}$ ) reveal quantum corrections to the conductivity in materials with moderate disorder, called weaklocalization and antilocalization. ${ }^{16-19}$ The quantum correction of weak-localization is observed in the measurements depicted in Figure 9b,c, as discussed below. Weak localization is a manifestation of the near-absence of spin-orbit interaction, in contrast to antilocalization which is a manifestation of the presence of spin-orbit interaction. ${ }^{18-22}$ The quantum corrections result from quantum interference of time-reversed closed-loop scattering trajectories. A negative magnetoresistance at low magnetic fields is a hallmark of weak-localization, whereas a positive magnetoresistance is a hallmark of antilocalization. $^{20}$ An initial decrease in resistance with increasing magnetic field (negative magnetoresistance, weaklocalization at low fields) is indeed observed in Figure 9b,c. The existence of weak-localization, and not antilocalization, points 
(a)

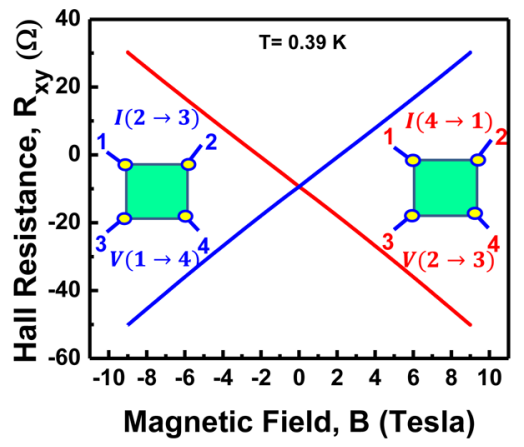

(b)

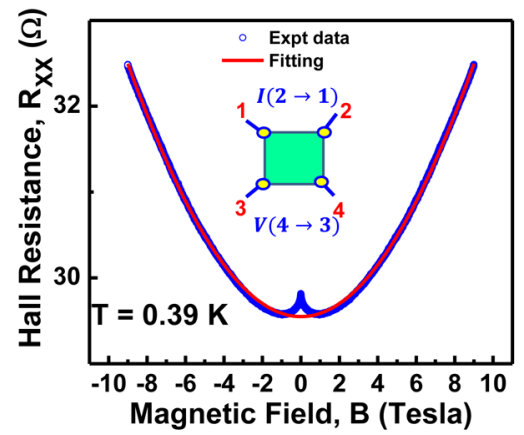

(c)

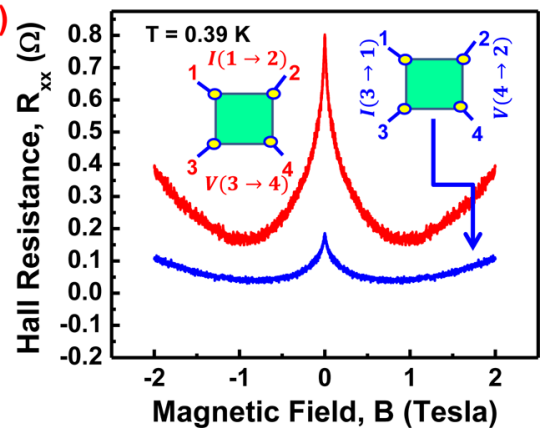

Figure 9. (a) Antisymmetric component of the Hall resistance, $R_{X Y}$ as a function of magnetic field over $\pm 9 \mathrm{~T}$, with the very linear dependence on magnetic field demonstrating single carrier behavior. (b) Symmetric component of the Hall resistance, $\mathrm{R}_{\mathrm{xx}}$ as a function of magnetic field over $\pm 9 \mathrm{~T}$. (c) High-resolution traces, with different resistance offsets subtracted, emphasizing the weak-localization behavior at low magnetic fields.

to the fact that the carriers responsible for transport experience a near absence of spin-orbit interaction. As explained below, in the Ge/AlAs heterostructure this observation indicates that the carriers responsible for transport are electrons confined to the Ge layer, and excludes transport contributions due to carriers in the AlAs layer. Figure $9 \mathrm{~b}, \mathrm{c}$ show the symmetric component of the Hall resistance (diagonal component $\mathrm{R}_{\mathrm{xx}}$ ) up to $\pm 9 \mathrm{~T}$ magnetic field with the different configurations of current and voltage contacts as depicted schematically in the insets for the van der Pauw geometry. Figure $9 \mathrm{~b}$ shows the weak-localization negative magnetoresistance at low field (below $\sim 1 \mathrm{~T}$ ). Figure 9c shows high-resolution traces at low fields (resistance offsets were subtracted during the measurement to obtain higher measurement resolutions). Figure $9 \mathrm{c}$ clearly shows the existence of the negative magnetoresistance characteristic of weak-localization. As mentioned above, the carriers responsible for transport thus must experience negligible spin-orbit interaction. Spin-orbit interaction occurs due to a broken spatial inversion symmetry, ${ }^{23}$ either in the bulk crystal (Dresselhaus effect ${ }^{24,25}$ ) or due to heterostructural asymmetry (Rashba effect ${ }^{26,27}$ ). The AlAs, as a III-V compound semiconductor, has a lack of inversion symmetry in the bulk crystal and thus carriers in AlAs necessarily experience spinorbit interaction, while in contrast, specifically electrons in $\mathrm{Ge}$ experience only negligible spin-orbit interaction. ${ }^{23-25}$ Thus, the transport in the Ge/AlAs heterostructure must occur by electrons only in the Ge layer. This confirms the single carrier conduction and pinpoints the Ge layer as the electronically active layer. The single carrier conduction and the detailed magnetotransport properties of the epitaxial Ge demonstrated above illustrate the strong potential for low-power transistors as well as optoelectronic devices.

\section{CONCLUSIONS}

The growth, structural, and magnetotransport properties of epitaxial $\mathrm{Ge} / \mathrm{AlAs}$ heterostructures with different growth conditions and substrate architectures have been studied under $\pm 9 \mathrm{~T}$ magnetic field and at $390 \mathrm{mK}$ temperature. Systematic mobility measurements of Ge epilayer on GaAs substrates with growth temperatures from 350 to $450{ }^{\circ} \mathrm{C}$ demonstrated the highest electron mobility at $400{ }^{\circ} \mathrm{C}$ without long growth pause prior to $\mathrm{Ge}$ deposition, which is corroborated by structural and morphological studies. Our results on $\mathrm{Si}$ substrate using a composite AlAs/GaAs buffer architecture at $400{ }^{\circ} \mathrm{C}$ Ge growth temperature demonstrates that the Ge/AlAs shows single carrier transport with the charge solely confined to the Ge layer. The prominent negative magnetoresistance at $390 \mathrm{mK}$ temperature is indicative of the weak-localization effect in this Ge/AlAs heterostructure and points to the near absence spin-orbit interaction, a first demonstration in this heterostructure indicating that carriers responsible for transport must be electrons confined to the $\mathrm{Ge}$ layer. Hence, epitaxial Ge grown on $\mathrm{Si}$ using an AlAs/GaAs buffer architecture shows a great promise for next-generation low-power and high-performance field effect transistor applications.

\section{MATERIALS AND METHODS}

Material Synthesis. The undoped epitaxial $80 \mathrm{~nm}-240 \mathrm{~nm}$ thick Ge layers were grown using an in situ growth process on epi-ready semi-insulating (100)/2 ${ }^{\circ}$ GaAs and (100)/ $6^{\circ}$ offcut Si substrates using separate solid source molecular beam epitaxy growth chambers for the $\mathrm{Ge}$ and III-V materials, connected via an ultrahigh vacuum transfer chamber. The growth temperature and growth rate of epitaxial Ge were in the range of $350-450{ }^{\circ} \mathrm{C}$ and $0.1 \AA / \mathrm{s}$, respectively. The details of the growth procedure are reported elsewhere. ${ }^{8,15}$

Materials Characterization. To determine the structural quality and the relaxation state of epitaxial Ge layers, we recorded highresolution triple axis $\mathrm{X}$-ray rocking curves. Cross-sectional highresolution transmission electron microscopy (HR-TEM) was used to characterize the interface between the Ge and AlAs as well as AlAs and GaAs substrate. The HR-TEM imaging was performed on a JEOL 2100 transmission electron microscope. For this purpose, the electron transparent foil of thin film cross-section of Ge/AlAs/GaAs was prepared by a standard polishing technique, that is, mechanical grinding, dimpling, and $\mathrm{Ar}^{+}$ion beam milling.

Carrier Transport Measurement. Au/Ti $(600 \AA / 200 \AA)$ ohmic contacts required for the Hall mobility measurements were made on $\mathrm{Ge} / \mathrm{AlAs} / \mathrm{GaAs}$ and Ge/AlAs/GaAs/Si in a Kurt J. Lesker PVD 250 physical vapor deposition system. The four corner contacts were defined using positive photoresist and prebaked at $\sim 85^{\circ} \mathrm{C}$ prior to the deposition of $\mathrm{Au}$ and Ti metals. The deposited contacts were annealed at $350{ }^{\circ} \mathrm{C}$ for 5,15 , and 25 min under a mixture of $\mathrm{N}_{2} / \mathrm{H}_{2}(95: 5 \mathrm{v} / \mathrm{v})$. 
The carrier density and Hall mobility were measured as a function of temperature from 90 to $315 \mathrm{~K}$ with a fixed magnetic field of $0.55 \mathrm{~T}$ using an Ecopia HMS5000 Hall measurement system. The magnetotransport measurements at $390 \mathrm{mK}$, and high magnetic fields were performed in a ${ }^{3} \mathrm{He}$ cryostat, with the sample submerged in liquid ${ }^{3} \mathrm{He}$. The ${ }^{3} \mathrm{He}$ system is equipped with a superconducting magnet allowing the magnetic field to be varied over $9 \mathrm{~T}$ in both polarities normal to the sample surface. The weak localization data was obtained in the same system using a magnet power supply capable of slow sweeps with subgauss resolution.

\section{AUTHOR INFORMATION}

\section{Corresponding Author}

*Tel: (540) 231-6663. Fax: (540) 231-3362. E-mail: mantu. hudait@vt.edu.

\section{Notes}

The authors declare no competing financial interest.

\section{ACKNOWLEDGMENTS}

This work was supported in part by Intel Corporation. M.C. acknowledges the financial support from NSF under grant number ECCS-1348653. P.G. acknowledges support from an NSF Graduate Research Fellowship. J.J.H. and Y.X. acknowledge support by the U.S. Department of Energy, Office of Basic Energy Sciences, Division of Materials Sciences and Engineering under award DOE DE-FG02-08ER46532. Authors also acknowledge NCFL-Institute for Critical Technology and Applied Science and Virginia Tech Nanofabrication facilities for materials characterization and contact formation, respectively.

\section{REFERENCES}

(1) Krishnamohan, T.; Krivokapic, Z.; Uchida, K.; Nishi, Y.; Saraswat, K. C. High-Mobility Ultrathin Strained Ge MOSFETs on Bulk and SOI With Low Band-to-Band Tunneling Leakage: Experiments. IEEE Trans. Electron Devices 2006, 53, 990-999.

(2) Krishnamohan, T.; Kim, D.; Nguyen, C. D.; Jungemann, C.; Nishi, Y.; Saraswat, K. C. High-Mobility Low Band-to-Band Tunneling Strained-Germanium Double-gate Heterostructure FETs: Simulations. IEEE Trans. Electron Devices 2006, 53, 1000-1009.

(3) Ho, B.; Nuo, X.; Tsu-Jae King, L. pMOSFET Performance Enhancement With Strained $\mathrm{Si}_{1-\mathrm{x}} \mathrm{Ge}_{\mathrm{x}}$ Channels. IEEE Trans. Electron Devices 2012, 59, 1468-1474.

(4) Chleirigh, C. N.; Theodore, N. D.; Fukuyama, H.; Mure, S.; Ehrke, H.-U.; Domenicucci, A.; Hoyt, J. L. Thickness Dependence of Hole Mobility in Ultrathin SiGe-channel p-MOSFETs. IEEE Trans. Electron Devices 2008, 55, 2687-2694.

(5) Hutin, L.; Le Royer, C.; Damlencourt, J.-F.; Hartmann, J.-M.; Grampeix, H.; Mazzocchi, V.; Tabone, C.; Previtali, B.; Pouydebasque, A.; Vinet, M. GeOI pMOSFETs Scaled Down to 30-nm Gate Length With Record Off-state Current. IEEE Electron Device Lett. 2010, 31, 234-236.

(6) Pillarisetty, R.; Chu-Kung, B.; Corcoran, S.; Dewey, G.; Kavalieros, J.; Kennel, H.; Kotlyar, R.; Le, V.; Lionberger, D.; Metz, M.; et al. High Mobility Strained Germanium Quantum Well Field Effect Transistor as the p-Channel Device Option for Low Power $\left(\mathrm{V}_{\mathrm{cc}}\right.$ $=0.5 \mathrm{~V})$ III-V CMOS Architecture. Technical Digest International Electron Devices Meeting-IEDM 2010, 6.7.1-6.7. 4.

(7) Zhang, R.; Yu, X.; Takenaka, M.; Takagi, S. Impact of Channel Orientation on Electrical Properties of Ge p- and n-MOSFETs With 1nm EOT $\mathrm{Al}_{2} \mathrm{O}_{3} / \mathrm{GeO}_{\mathrm{x}} / \mathrm{Ge}$ Gate-Stacks Fabricated by Plasma Post oxidation. IEEE Trans. Electron Devices 2014, 61, 3668-3675.

(8) Hudait, M. K.; Clavel, M.; Goley, P.; Jain, N.; Zhu, Y. Heterogeneous Integration of Epitaxial $\mathrm{Ge}$ on $\mathrm{Si}$ using AlAs/GaAs Buffer Architecture: Suitability for Low-power Fin Field-Effect Transistors. Sci. Rep. 2014, 4, 6964-6969.
(9) Nguyen, P. D.; Clavel, M.; Goley, P.; Liu, J.-S.; Allen, N.; Guido, L. J.; Hudait, M. K. Heteroepitaxial Ge MOS Devices on Si Using Composite AlAs/GaAs Buffer. IEEE J. Electron Devices Soc. 2015, 3, 341-348.

(10) Hudait, M. K. Heterogeneously Integrated III-V on Silicon for Future Nanoelectronics,. ECS Trans. 2012, 45, 581-594.

(11) Hudait, M. K.; Dewey, G.; Datta, S.; Fastenau, J. M.; Kavalieros, J.; Liu, W. K.; Lubyshev, D.; Pillarisetty, R.; Rachmady, W.; Radosavljevic, M.; Rakshit, T.; Chau, R. Heterogeneous Integration of Enhancement Mode $\mathrm{In}_{0.7} \mathrm{Ga}_{0.3}$ As Quantum Well Transistor on Silicon Substrate Using Thin $(2 \mu \mathrm{m})$ Composite Buffer Architecture for High-speed and Low-voltage (0.5V) Logic Applications. IEEE International Electron Devices Meeting (IEDM) Technical Digest 2007, 625-628.

(12) Lubyshev, D.; Fastenau, J. M.; Wu, Y.; Liu, W. K.; Bulsara, M. T.; Fitzgerald, E. A.; Hoke, W. E. Molecular Beam Epitaxy Growth of Metamorphic High Electron Mobility Transistors and Metamorphic Heterojunction Bipolar Transistors on $\mathrm{Ge}$ and Ge-on-Insulator/Si Substrates. J. Vac. Sci. Technol. B 2008, 26, 1115-1119.

(13) Hudait, M. K.; Lin, Y.; Sinha, P. M.; Lindemuth, J. R.; Ringel, S. A. Carrier Compensation and Scattering Mechanisms in Si-doped InAs $\mathrm{P}_{1-\mathrm{y}}$ Layers Grown on InP Substrates Using Intermediate InAs $\mathrm{P}_{1-\mathrm{y}}$ step-Graded buffers. J. Appl. Phys. 2006, 100, 063705-1-9.

(14) Lindemuth, J. R. Parallel Conduction in Semiconductors. III-Vs Review 2006, 19, 28-32.

(15) Hudait, M. K.; Zhu, Y.; Jain, N.; Hunter, J. L., Jr. Structural, Morphological, and Band Alignment Properties of GaAs/Ge/GaAs Heterostructures on (100), (110) and (111)A GaAs Substrates. J. Vac. Sci. Technol. B 2013, 31, 011206-1-14.

(16) Bergmann, G. Weak Localization and its Applications as an Experimental Tool. Int. J. Mod. Phys. B 2010, 24, 2015-2052.

(17) Bergmann, G. Weak Localization in Thin Films, a Time-of-flight Experiment With Conduction Electrons. Phys. Rep. 1984, 107, 1-58.

(18) Kallaher, R. L.; Heremans, J. J. Spin and Phase Coherence Measured by Antilocalization in $\mathrm{n}-\mathrm{InSb}$ Thin Films. Phys. Rev. B: Condens. Matter Mater. Phys. 2009, 79, 075322.

(19) Kallaher, R. L.; Heremans, J. J.; Goel, N.; Chung, S. J.; Santos, M. B. Spin-orbit Interaction Determined by Antilocalization in an InSb Quantum Well. Phys. Rev. B: Condens. Matter Mater. Phys. 2010, 81, 075303.

(20) Bergmann, G. Weak Anti-localization - An Experimental Proof for the Destructive Interference of Rotated Spin 1/2. Solid State Commun. 1982, 42, 815-817.

(21) Iordanskii, S. V.; Lyanda-Geller, Y. B.; Pikus, G. E. Weak Localization in Quantum Wells With Spin-orbit Interaction. JETP Lett. 1994, 60, 206-211.

(22) McPhail, S.; Yasin, C. E.; Hamilton, A. R.; Simmons, M. Y.; Linfield, E. H.; Pepper, M.; Ritchie, D. A. Weak Localization in Highquality Two-dimensional Systems. Phys. Rev. B: Condens. Matter Mater. Phys. 2004, 70, 245311.

(23) Winkler, R. Spin-orbit Coupling Effects in Two-dimensional Electron and Hole Systems. Springer Tracts in Modern Physics; Springer-Verlag: Berlin, Heidelberg, 2003.

(24) Dresselhaus, G. F. Spin-orbit Coupling Effects in Zinc Blende Structures. Phys. Rev. 1955, 100, 580-586.

(25) Faniel, S.; Matsuura, T.; Mineshige, S.; Sekine, Y.; Koga, T. Determination of Spin-Orbit Coefficients in Semiconductor Quantum Wells. Phys. Rev. B: Condens. Matter Mater. Phys. 2011, 83, 115309.

(26) Bychkov, Y. A.; Rashba, E. I. Oscillatory Effects and the Magnetic Susceptibility of Carriers in Inversion Layers. J. Phys. C: Solid State Phys. 1984, 17, 6039-6045.

(27) Pfeffer, P.; Zawadzki, W. Spin Splitting of Conduction Subbands in III-V Heterostructures Due to Inversion Asymmetry. Phys. Rev. B: Condens. Matter Mater. Phys. 1999, 59, R5312-R5315. 